\title{
Incorporación de materiales de cambio de fase en placas de yeso para almacenamiento de energía térmica mediante calor latente: caracterización térmica del material mediante la técnica DSC
}

\author{
PCM inclusion in gypsum boards for thermal energy storage \\ through latent heat: thermal characterization with DSC
}

\author{
$\underline{\text { A. Oliver }}^{(*)}$, F. J. Neila ${ }^{(*)}$, A. García ${ }^{(*)}$
}

\section{RESUMEN}

La Calorimetría Diferencial de Barrido es una técnica de análisis térmico, usada desde hace décadas, para medir la entalpía asociada al cambio de fase de un material como función del tiempo y de la temperatura. Otras técnicas menos utilizadas son la Calorimetría Convencional el Análisis Térmico Diferencial.

Existe una gran incertidumbre en los valores de propiedades suministrados por los fabricantes (puesto que éstos se refieren a las sustancias puras) y es conveniente utilizar DSC para tener valores más exactos.

Se va a analizar la capacidad de almacenamiento térmico en función de la temperatura de varios materiales compuestos formados por los mismos agregados -principalmente yeso y material de cambio de fase- en distintas proporciones. Los valores obtenidos se comparan con otros materiales constructivos, yeso laminado y ladrillo.

También se verifica la idoneidad del nuevo material constructivo para el almacenamiento de energía térmica frente a otros materiales utilizados tradicionalmente para este fin.

$310-14$

Palabras clave: calorimetría diferencial de barrido, material de cambio de fase, entalpía, energía térmica, yeso.

\section{SUMMARY}

Differential Scanning Calorimetry (DSC) is a thermal analysis technique which has been used for more than three decades to measure the temperatures and heat flows associated with transitions in materials as a function of time. Other techniques, are Differential Thermal Analysis DTA and Conventional Calorimetry.

There is great uncertainty in the values supplied by the manufacturers (because they are referred to pure substances) and the DSC should be used to get more accurate values.

It will be analyzed the thermal storage capacity depending on temperature for several compound materials formed by some aggregates, mainly gypsum and phase change materials, in various proportions. The results have been compared with other building materials such as gypsum boards and brick layer.

The suitability of the new construction material for thermal energy storage will be assessed in comparison with other materials traditionally used for this purpose.

Keywords: DSC, PCM, enthalpy, thermal energy, gypsum. 


\section{ANTECEDENTES}

La Calorimetría Diferencial de Barrido (del inglés: Differential Scanning Calorimetry o DSC) es una técnica termoanalítica en la que la diferencia de calor entre una muestra y una referencia es medida como una función de la temperatura. El resultado de un experimento DSC es una curva de flujo calorífico versus temperatura o versus tiempo.

Una técnica alternativa a la DSC es el Análisis Térmico Diferencial (DTA). En esta técnica la magnitud constante no es la temperatura sino el flujo calorífico que se comunica a muestra y referencia. Ambas DSC y DTA proporcionan, pues, información similar siendo la DSC es más utilizada que la DTA.

Existen dos convenciones diferentes al representar los efectos térmicos: las reacciones exotérmicas que exhibe la muestra pueden ser mostradas como picos positivos o negativos dependiendo del tipo de tecnología o de instrumentación utilizadas en la realización del experimento. Los efectos sobre o bajo una curva DSC pueden ser utilizados para calcular entalpías de transiciones. Este cálculo se realiza integrando el pico correspondiente a una transición dada. Así, la entalpía de la transición puede ser expresada por la siguiente ecuación: $\Delta \mathrm{H}=\mathrm{KA}$.

Donde $\Delta \mathrm{H}$ es la entalpía de la transición, $\mathrm{K}$ es la constante calorimétrica y $\mathrm{A}$ es el área bajo la curva. La constante calorimétrica variará de instrumento a instrumento, y puede ser determinada analizando una muestra bien caracterizada con entalpías de transición conocidas.

Los materiales de cambio de fase (PCM, Phase Change Materials) son utilizados desde hace décadas, en el campo de la construcción entre otros, para almacenamiento de energía térmica.

Numerosos autores (1-4), han estudiado sus propiedades físicas, químicas, cinéticas, y especialmente las térmicas, a partir de las cuales podremos elegir el material más adecuado a nuestros propósitos.

En el campo de la construcción existen diversas publicaciones que estudian el comportamiento térmico de materiales y sistemas constructivos que incorporan PCM (5-8).

En este trabajo se ha utilizado yeso como material constructivo para combinar con PCM, por su gran disponibilidad, bajo coste, y profusa utilización en construcción. Existen diversos trabajos que estudian el comportamiento térmico de placas de yeso con PCM incorporado. Unos lo estudian con la técnica DSC, como (9-11) para conocer las propie- dades intrínsecas del nuevo material compuesto, y otros analizan el comportamiento térmico del elemento constructivo -con unas dimensiones una forma de presentación y una posición determinada-a escala real, es decir, instalado en una habitación experimental como (12-15).

A partir de los resultados obtenidos pueden obtenerse modelos matemáticos de comportamiento térmico del nuevo elemento constructivo, como se ha hecho en los trabajos presentados por (16-19).

\section{EXPERIMENTAL}

En este trabajo el material utilizado en este trabajo es una parafina microencapsulada disponible comercialmente: Micronal DS 5001X. El único dato ofrecido por el fabricante es la temperatura de cambio de fase, del que además se sabe que, por tratarse de un compuesto orgánico, el cambio de estado se produce en un rango de temperaturas, no para un valor determinado, con una relación temperatura-entalpía desconocida.

En este estudio se va a analizar con el método DSC la entalpía de un compuesto formado por yeso y material de cambio de fase en una relación 1/0,8 entre ambos, es decir un $44,5 \%$ en peso de PCM. Esto supone un gran incremento en el porcentaje incorporado de PCM a una placa de yeso ya que en toda la bibliografía consultada la máxima cantidad incorporada es de un $26 \%$.

Para poder incorporar esta gran cantidad de PCM -conservando sus propiedades físicas y mecánicas- se han elegido materiales de refuerzo, basado en trabajos anteriores (20) los cuales se han combinado en diferentes dosis para obtener el compuesto ideal, es decir, aquel que conteniendo mayor cantidad de PCM, cumple con las recomendaciones establecidas en la normativa de aplicación.

Se han elegido cuatro compuestos representativos por su composición, para examinarlos mediante la técnica de DSC a fin de conocer las características térmicas, el proceso de fusión y solidificación en este intervalo de temperaturas.

Todas las muestras se han sometido a un programa de calentamiento de 5 a $45{ }^{\circ} \mathrm{C}$ a $5^{\circ} \mathrm{C} / \mathrm{min}$ seguido de un enfriamiento de 45 a $5{ }^{\circ} \mathrm{C}$ también a $5{ }^{\circ} \mathrm{C} / \mathrm{min}$, que es un rango de temperatura algo mayor que el que se usa normalmente en construcción, de $15-30{ }^{\circ} \mathrm{C}$.

Aunque esta velocidad de calentamiento/ enfriamiento no se corresponde con el ciclo día/noche, estos ensayos muestran el calor total asociado, y el equipo permite definir las 
velocidades en un intervalo muy amplio que va desde 0,0001 hasta $300^{\circ} \mathrm{C} / \mathrm{min}$.

El comportamiento térmico de los paneles de yeso con PCM incorporado a escala real se estudia en (21). En este trabajo se ha diseñado y construido un circuito experimental para realizar ensayos con los paneles. Éstos se colocan en una caja intercambiadora de dimensiones $0,80 \times 0,60 \times 0,60 \mathrm{~m}^{3}$ y se mide la variación de la temperatura de éstos y del ambiente interior en el tiempo al hacer pasar un flujo de aire con una velocidad y una temperatura determinada.

La confección de paneles de yeso reforzados y parafinas microencapsuladas se ha realizado por adición directa, procurando que la dosis de cada agregado sea la adecuada para incorporar la máxima cantidad de PCM manteniendo las propiedades físicas y mecánicas del yeso.

Las cuatro sustancias elegidas son (Tabla1, y ver Figura 1).

\section{ANÁLISIS DE RESULTADOS}

\subsection{Muestra $Q$}

En esta muestra, que se realiza con yeso, no se observa un pico de fusión significativo, por lo que la mayor parte del calor almacenado es debido a su capacidad calorífica, es decir, al calor sensible asociado a la sustancia. En efecto, el único material que no posee $\mathrm{PCM}$.

Para los 10,867 g de sustancia analizados se ha necesitado una energía, $\Delta \mathrm{H}=\mathrm{Q} s=720,08$ $\mathrm{mJ}$, es decir $66,26 \mathrm{~J} / \mathrm{g}$, para elevarla $45^{\circ} \mathrm{C}$, es decir $1,47 \mathrm{~J} / \mathrm{g}{ }^{\circ} \mathrm{C}$, superior al $1,00 \mathrm{~J} / \mathrm{g}{ }^{\circ} \mathrm{C}$ que posee la yeso pura como calor específico. Ello se puede explicar por la presencia de fibras de polipropileno, cuyo calor específico es del orden de 1,45 J/g.K, -aunque sólo es un $2 \%$ - y porque los valores tabulados son genéricos del material, nunca específicos (Ver Figura 2 y Figura 3).

En la Tabla 2 se calculan los valores obtenidos a partir de los resultados del ensayo -representados en Gráfica 1- de acuerdo con la Ecuación [1]

$$
\mathrm{Qs}=\mathrm{m}(\mathrm{g}) \cdot \mathrm{ce}(\mathrm{J} / \mathrm{g} \cdot \mathrm{K}) \cdot \Delta \mathrm{T}\left({ }^{\circ} \mathrm{C}\right),
$$

donde Qs, energía almacenada por calor sensible, $\mathrm{m}$, y ce, son la masa y el calor específico del material (Tabla 2), Esta Gráfica 1 no es totalmente trapezoidal, como sería de esperar, donde la integral de la pendiente sería una constante correspondiente al calor específico de la sustancia. Ello se debe a que no es una sustancia pura, sino que tiene ciertas impurezas -fibras de polipropileno-.

Hay que señalar que la velocidad de cambio de temperatura a la que se ha hecho este ensayo es muy superior a la que se da en condiciones reales (para una variación máxima de temperatura entre el día y la noche de $19-20{ }^{\circ} \mathrm{C}$, lo que supone $1,6^{\circ} \mathrm{C} / \mathrm{h}$ ), por lo que es posible que los resultados se vean influenciados por las cuestiones cinéticas de
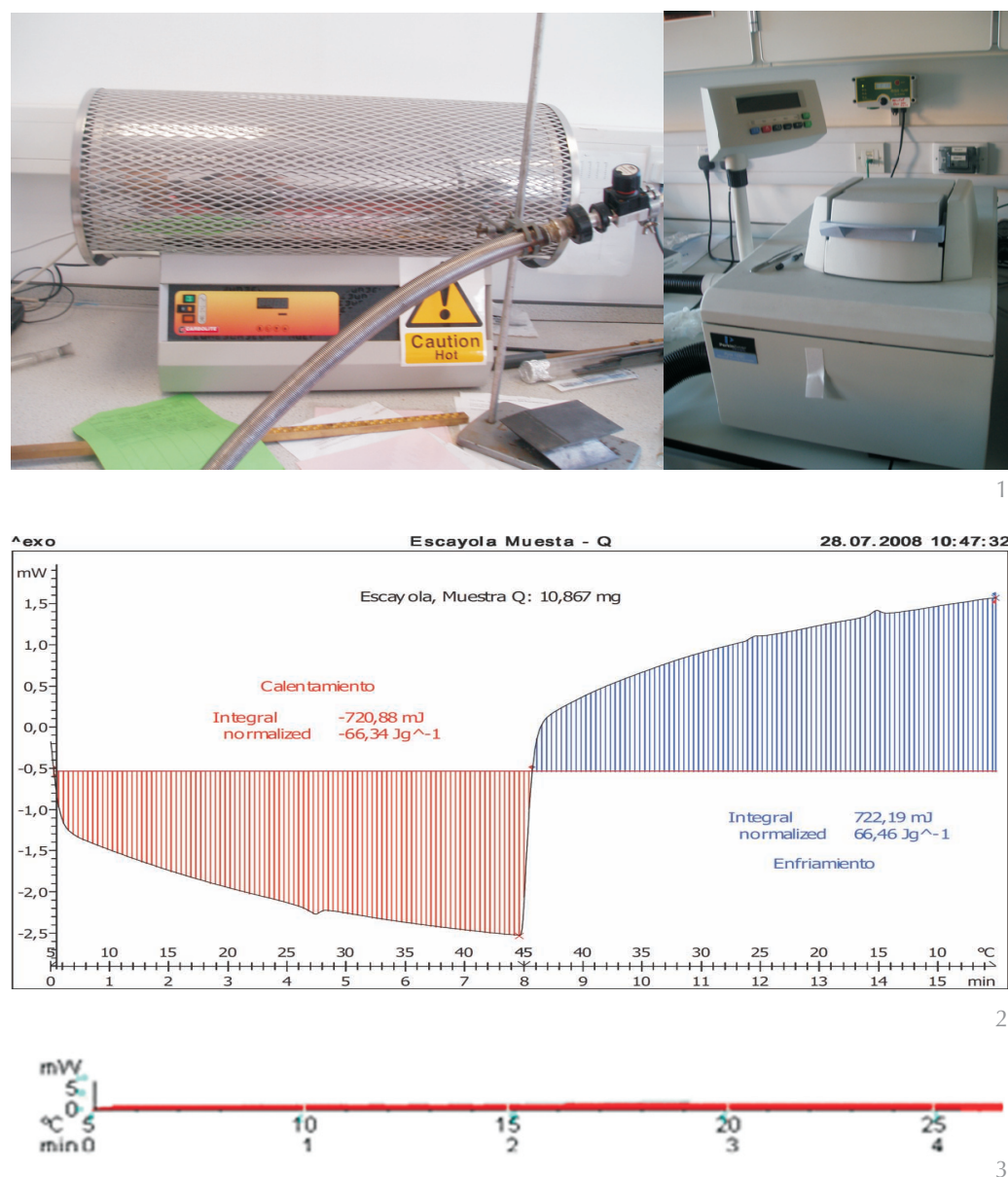

Tabla 1

Proporción de los diferentes agregados en cada una de las sustancias respecto a la masa de yeso

\begin{tabular}{|c|c|c|c|c|c|}
\hline MUESTRA & yeso & agua & fibra & dispersión & PCM \\
\hline S & 100,00 & 90,00 & 2,00 & 0,00 & 60,00 \\
\hline D & 100,00 & 100,00 & 3,50 & 4,00 & 80,00 \\
\hline C & 100,00 & 100,00 & 3,50 & 4,00 & 100,00 \\
\hline Q & 100,00 & 90,00 & 2,00 & 0,00 & 0,00 \\
\hline
\end{tabular}

Tabla 2

Valores obtenidos del ensayo de la sustancia Q

\begin{tabular}{|l|c|c|c|c|c|c|c|}
\hline \multicolumn{1}{|c|}{$\mathbf{M}$} & $\begin{array}{c}\text { Masa } \\
(\mathbf{m g})\end{array}$ & $\begin{array}{c}\text { Qlat } \\
(\mathbf{m} \mathbf{)})\end{array}$ & $\begin{array}{c}\mathbf{Q}_{\text {lat }} / \mathbf{M} \\
(\mathbf{m} \mathbf{j} / \mathbf{g})\end{array}$ & $\begin{array}{c}\mathbf{Q}_{\text {sen }} \\
(\mathbf{m J})\end{array}$ & $\begin{array}{c}\mathbf{Q}_{\text {sen }} / \mathbf{M} \\
\left(\mathbf{m} \mathbf{j} / \mathbf{g}^{\circ} \mathbf{C}\right)\end{array}$ & $\begin{array}{c}\mathbf{Q}_{\text {lat }}+\mathbf{Q}_{\text {sen }} \\
(\mathbf{m} \mathbf{)})\end{array}$ & $\begin{array}{c}\Delta \mathbf{H} / \mathbf{M} \\
(\mathbf{m J} / \mathbf{g})\end{array}$ \\
\hline PCM & 0,00 & 0,00 & 0,00 & 0,00 & 0,00 & 0,00 & 0,00 \\
\hline Yeso & 10,87 & 0,00 & 0,00 & 720,08 & 1,47 & 720,08 & 66,26 \\
\hline yeso + PCM & 10,87 & 0,00 & 0,00 & 720,08 & 1,47 & 720,08 & 66,26 \\
\hline
\end{tabular}




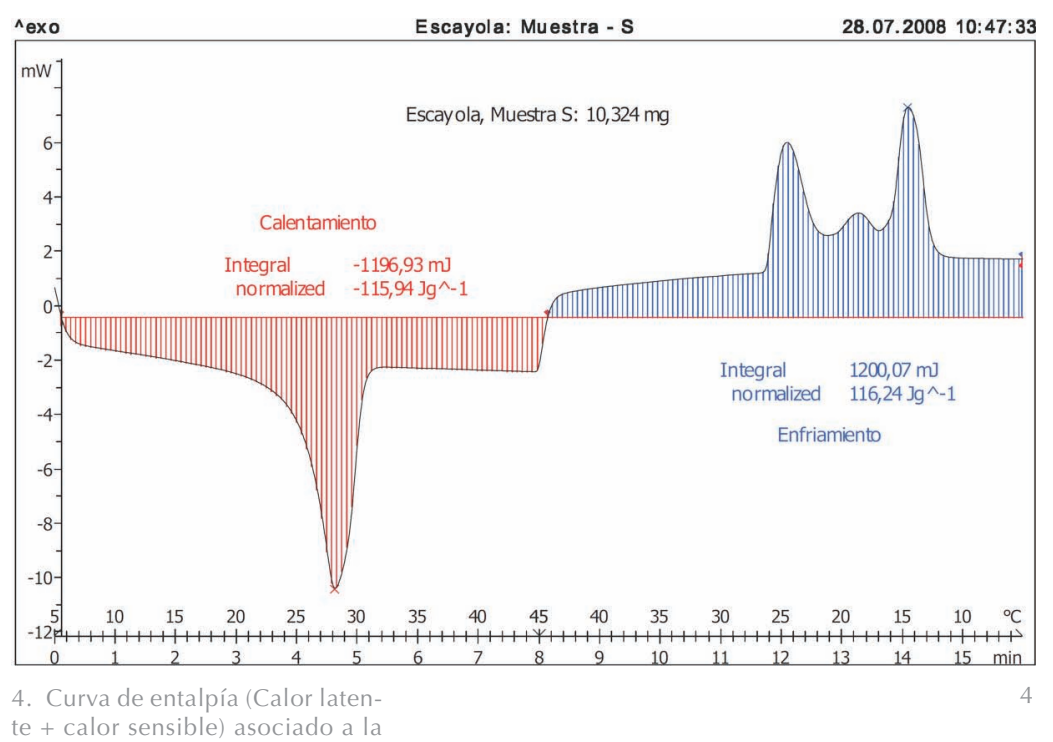

sustancia $S$.

Tabla 3

Valores obtenidos del ensayo de la sustancia S

\begin{tabular}{|c|r|r|r|r|r|r|r|}
\hline $\mathbf{M}$ & $\begin{array}{c}\text { Masa } \\
(\mathbf{m g})\end{array}$ & $\begin{array}{c}\text { Qlat } \\
(\mathbf{m} \mathbf{)})\end{array}$ & $\begin{array}{c}\mathbf{Q}_{\text {lat }} / \mathbf{M} \\
(\mathbf{m} \mathbf{j} / \mathbf{g})\end{array}$ & $\begin{array}{c}\mathbf{Q}_{\text {sen }} \\
(\mathbf{m} \mathbf{)})\end{array}$ & $\begin{array}{c}\mathbf{Q}_{\text {sen }} / \mathbf{M} \\
\left(\mathbf{m} / \mathbf{g}^{\circ} \mathbf{C}\right)\end{array}$ & $\begin{array}{c}\mathbf{Q}_{\text {lat }}+\mathbf{Q}_{\text {sen }} \\
(\mathbf{m} \mathbf{)})\end{array}$ & $\begin{array}{c}\Delta \mathbf{H} / \mathbf{M} \\
(\mathbf{m} \mathbf{J} / \mathbf{g})\end{array}$ \\
\hline PCM & 3,87 & 411,65 & 106,33 & 357,85 & 2,05 & 769,50 & 198,76 \\
\hline Yeso & 6,45 & 0,00 & 0,00 & 427,43 & 1,47 & 427,43 & 66,24 \\
\hline Yeso + PCM & 10,32 & 411,65 & $1.063,28$ & 785,28 & 1,69 & $1.196,93$ & 115,94 \\
\hline
\end{tabular}

formación de cristales que intervienen en este proceso de fusión-solidificación.

El proceso de fusión y solidificación son casi simétricos, aunque no exactamente iguales, lo que viene a demostrar que la sustancia que se está analizando contiene ciertas impurezas, que alteran el proceso de formación de cristales propio de una sustancia pura. Así nos lo muestra la siguiente gráfica obtenida.

La representación de los resultados en una gráfica, donde los valores de ordenadas (tiempo en segundos) tengan la misma escala que los de abscisas, (flujo en $\mathrm{mW}$ ), de forma que el área se

5. Calor latente asociado a la sustancia S.

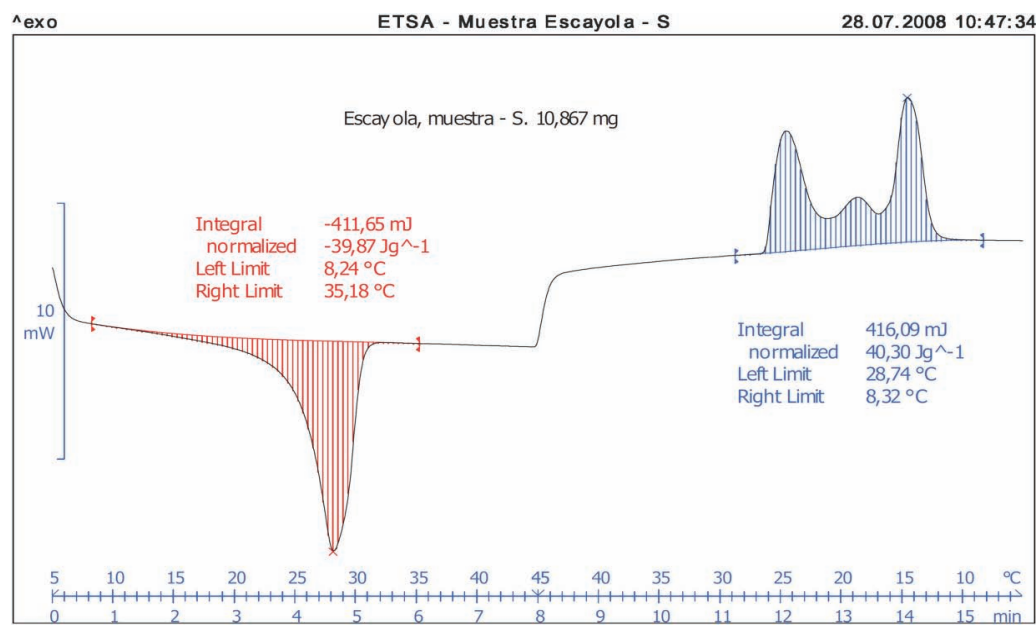

corresponda con la energía aportada (mJ), nos describe un trapecio sin apenas pendiente en el tiempo: Figura 3.

\subsection{Muestra S}

La siguiente sustancia analizada es la $S$, con igual dosificación que la $\mathrm{Q}$, salvo en el contenido de PCM, que está en un $60 \%$ en peso en relación al $100 \%$ de yeso, es decir un 37\% respecto al peso total del compuesto.

En la Figura 4 se observa con claridad los efectos producidos al incorporar un material de cambio de fase, ya que aparece un pico en la temperatura próxima al cambio de estado del material, y la energía necesaria para producir el cambio es muy elevada.

El gráfico correspondiente al calor sensible asociado al material compuesto (yeso + PCM) apenas ha variado respecto al del yeso puro. Se tiene que para 10,324 mg de sustancia analizada -de los cuales 3,871 corresponden a PCM- el calor latente es de 411,65 mJ y el sensible es de 785,28 mJ. Hay que tener en cuenta que la variación de temperatura es muy grande $\left(45^{\circ} \mathrm{C}\right)$, por lo que el valor del calor sensible, directamente dependiente de este parámetro también es elevado.

La Tabla 3 de valores obtenida en este ensayo es (ver Tabla3):

Además se ha obtenido la temperatura inferior a la que se ha iniciado el cambio de fase, $8,24{ }^{\circ} \mathrm{C}$, y la del final de cambio de fase, $35,18^{\circ} \mathrm{C}$. Este rango de temperatura o ventana térmica, es muy amplio, y complementa los datos aportados por el fabricante, que solamente establece una temperatura teórica de cambio de fase en $26^{\circ} \mathrm{C}$, algo diferente de la obtenida en este ensayo, más próximo a $28^{\circ} \mathrm{C}$.

En la Figura 5 se muestra el valor del calor latente del material.

En este caso el calor latente 411,65 mJ, para 4,075 g supone una tasa de 106,32 J/g.

Si se compara el proceso de fusión con el de solidificación, se observa que no son simétricos, en cuanto a calor latente, o cambio de fase se refiere. Sí ocurre, en el caso del calor sensible, como se ha visto también en el caso anterior.

Se observan dos picos de temperatura de vitrificación más acusados y uno más suave entre ellos a 25 y a $15^{\circ} \mathrm{C}$, y $18,5^{\circ} \mathrm{C}$, respectivamente. Siendo además el rango de temperatura de vitrificación entre $28,74{ }^{\circ} \mathrm{C}$ y $8,32{ }^{\circ} \mathrm{C}$, ligeramente diferente al de fusión.

La Figura 6 muestra el grado de conversión expresado de 0 a $100 \%$ en el intervalo de temperatura de 5 a $45{ }^{\circ} \mathrm{C}$. 
Tabla 4

Valores obtenidos del ensayo de la sustancia D

\begin{tabular}{|l|c|r|r|c|c|c|c|}
\hline \multicolumn{1}{|c|}{$\mathbf{M}$} & $\begin{array}{c}\text { Masa } \\
(\mathbf{m g})\end{array}$ & \multicolumn{1}{c|}{$\begin{array}{c}\text { Qlat } \\
(\mathbf{m} \mathbf{)})\end{array}$} & $\begin{array}{c}\mathbf{Q}_{\text {lat }} / \mathbf{M} \\
(\mathbf{m} \mathbf{j} / \mathbf{g})\end{array}$ & $\begin{array}{c}\mathbf{Q}_{\text {sen }} \\
(\mathbf{m J})\end{array}$ & $\begin{array}{c}\mathbf{Q}_{\text {sen }} / \mathbf{M} \\
\left(\mathbf{m} / \mathbf{g}{ }^{\circ} \mathbf{C}\right)\end{array}$ & $\begin{array}{c}\mathbf{Q}_{\text {lat }}+\mathbf{Q}_{\text {sen }} \\
(\mathbf{m} \mathbf{)})\end{array}$ & $\begin{array}{c}\Delta \mathbf{H} / \mathbf{M} \\
(\mathbf{m} \mathbf{J} / \mathbf{g})\end{array}$ \\
\hline PCM & 3,67 & 381,30 & 104,02 & 338,80 & 2,05 & 720,10 & 196,46 \\
\hline Yeso & 4,57 & 0,00 & 0,00 & 302,83 & 1,47 & 302,83 & 66,24 \\
\hline Yeso + PCM & 8,24 & 381,30 & 104,02 & 641,64 & 1,73 & $1.022,93$ & 124,19 \\
\hline
\end{tabular}

6. Porcentaje de sustancia licuada o solidificada en función de la temperatura.

7. Comparación de las curvas de entalpía de las sustancias Q y S

8. Curva de entalpía asociada a la sustancia D.
Se observa que estas dos funciones tampoco son simétricas, ni lineales, sino que presentan irregularidades, propia de materiales orgánicos. En estas condiciones de ensayo se observa que para un rango de temperaturas de trabajo entre $18{ }^{\circ} \mathrm{C}$ y $35^{\circ} \mathrm{C}$, el porcentaje de PCM fundido es del $17,75 \%$, y del $80,27 \%$.

Es importante considerar este aspecto, a la hora de calcular la cantidad necesaria de este material para acondicionar un edificio.

Comparando las dos gráficas Q, y S, (Gráfica 6) se observa que el calor sensible es muy similar en ambos casos, con ligeras variaciones debido a la introducción de una nueva sustancia en un $37,5 \%$ del total del peso, con un nuevo calor sensible asociado. Además las cantidades de compuesto analizado varían sensiblemente en uno y otro caso.

Si se considera el compuesto como una sustancia única, el calor específico que se obtiene es de 1,605 J/g.K, más elevado que en el primer caso, sin PCM, donde se obtenía 1,47 $\mathrm{J} / \mathrm{g} \cdot \mathrm{K}$. Ello indicaría que el calor específico del Micronal, es mucho más elevado, del orden de $2,054 \mathrm{~J} / \mathrm{g} \cdot \mathrm{K}$, lo cual varía sensiblemente respecto a los datos suministrados por el fabricante $(1,2 \mathrm{~J} / \mathrm{g} \cdot \mathrm{K})$.

Para poder hacer una comparativa de los resultados del ensayo, se ha repetido para las muestras $C$ y $D$, en las que varía la dosis de PCM obteniendo los resultados que se reflejan a continuación en Figura 7.

\subsection{Muestra D}

La muestra D, contiene un $80 \%$ en peso de Micronal (un $44,7 \%$ respecto al peso total del compuesto). Es la que se ha elegido para desarrollar comercialmente, por sus características físicas y mecánicas, de acuerdo con la normativa, y por su alto contenido en PCM.

En este ensayo para 8,237 mg de material la entalpía asociada al proceso de fusión es de 1022,93 mJ, sensiblemente diferente a la del proceso de solidificación, donde es de $1024,10 \mathrm{~mJ}$. Los valores obtenidos se exponen en Tabla 4.

En la Figura 8, se representan los datos obtenidos en el ensayo. Se observa también una asimetría en el proceso de endurecimiento o so-
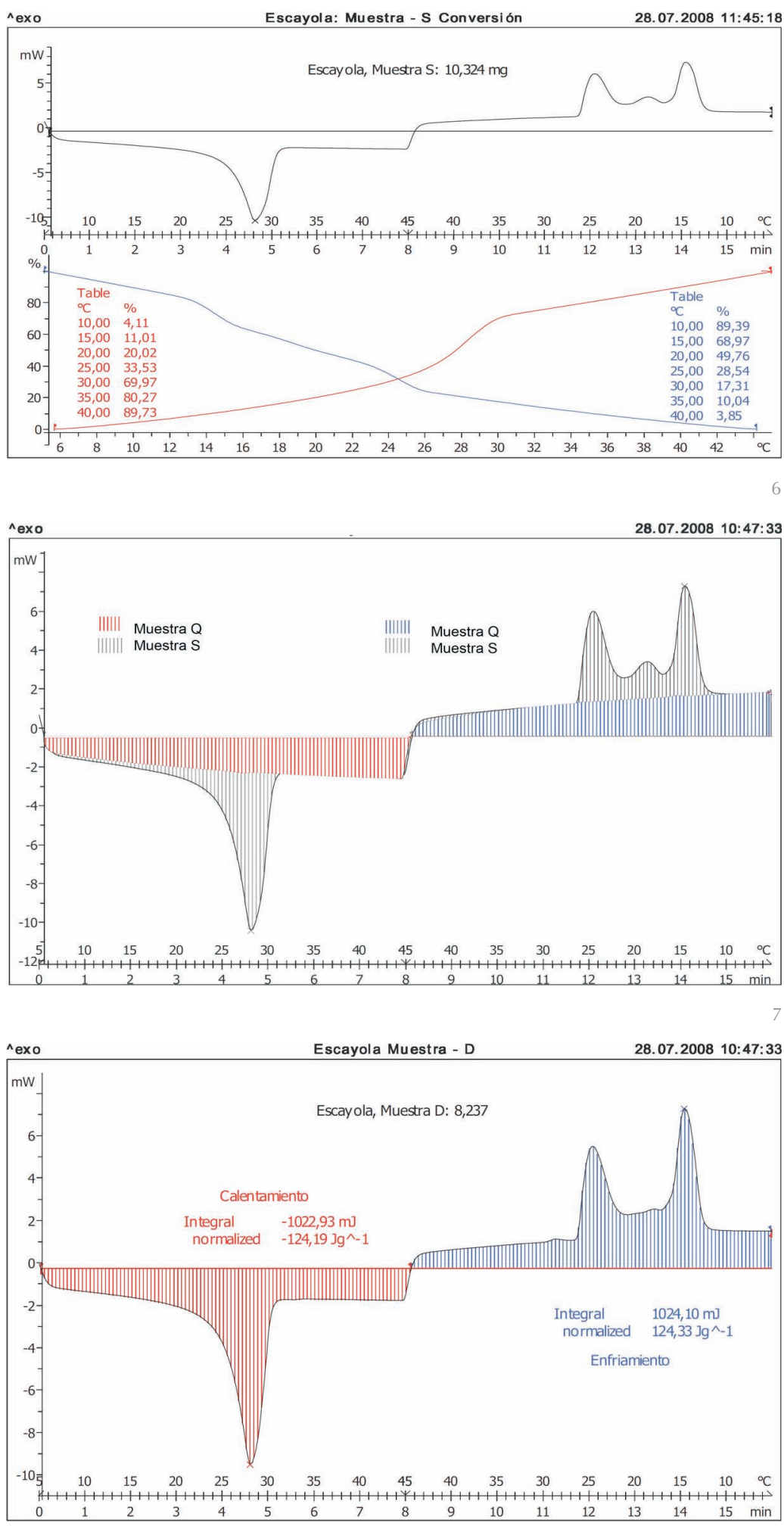
Tabla 5

Valores para la muestra $\mathrm{C}$

\begin{tabular}{|c|c|c|c|c|c|c|c|}
\hline $\mathbf{M}$ & $\begin{array}{c}\text { Masa } \\
(\mathbf{m g})\end{array}$ & $\begin{array}{c}\text { Qlat } \\
(\mathbf{m} \mathbf{)})\end{array}$ & $\begin{array}{c}\mathbf{Q}_{\text {lat }} / \mathbf{M} \\
(\mathbf{m} \mathbf{j} / \mathbf{g})\end{array}$ & $\begin{array}{c}\mathbf{Q}_{\text {sen }} \\
(\mathbf{m J})\end{array}$ & $\begin{array}{c}\mathbf{Q}_{\text {sen }} / \mathbf{M} \\
\left(\mathbf{m} / \mathbf{g}^{\circ} \mathbf{C}\right)\end{array}$ & $\begin{array}{c}\mathbf{Q}_{\text {lat }}+\mathbf{Q}_{\text {sen }} \\
(\mathbf{m} \mathbf{)})\end{array}$ & $\begin{array}{c}\mathbf{H} / \mathbf{M} \\
(\mathbf{m} \mathbf{J} / \mathbf{g})\end{array}$ \\
\hline PCM & 4,51 & 477,55 & 105,79 & 417,23 & 2,05 & 894,78 & 198,22 \\
\hline Yeso & 4,51 & 0,00 & 0,00 & 299,02 & 1,47 & 299,02 & 66,24 \\
\hline
\end{tabular}

lidificación respecto al de fusión, con dos picos muy marcados, a 25 y a $15{ }^{\circ} \mathrm{C}$, que coinciden con los obtenidos en la gráfica de la sustancia $\mathrm{S}$. En este ensayo el pico intermedio ha desaparecido es menos notorio. Ello refuerza la teoría de que la presencia de varias sustancias en el compuesto altera el proceso de solidificación.

9. Curva de entalpía asociada a la sustancia C.
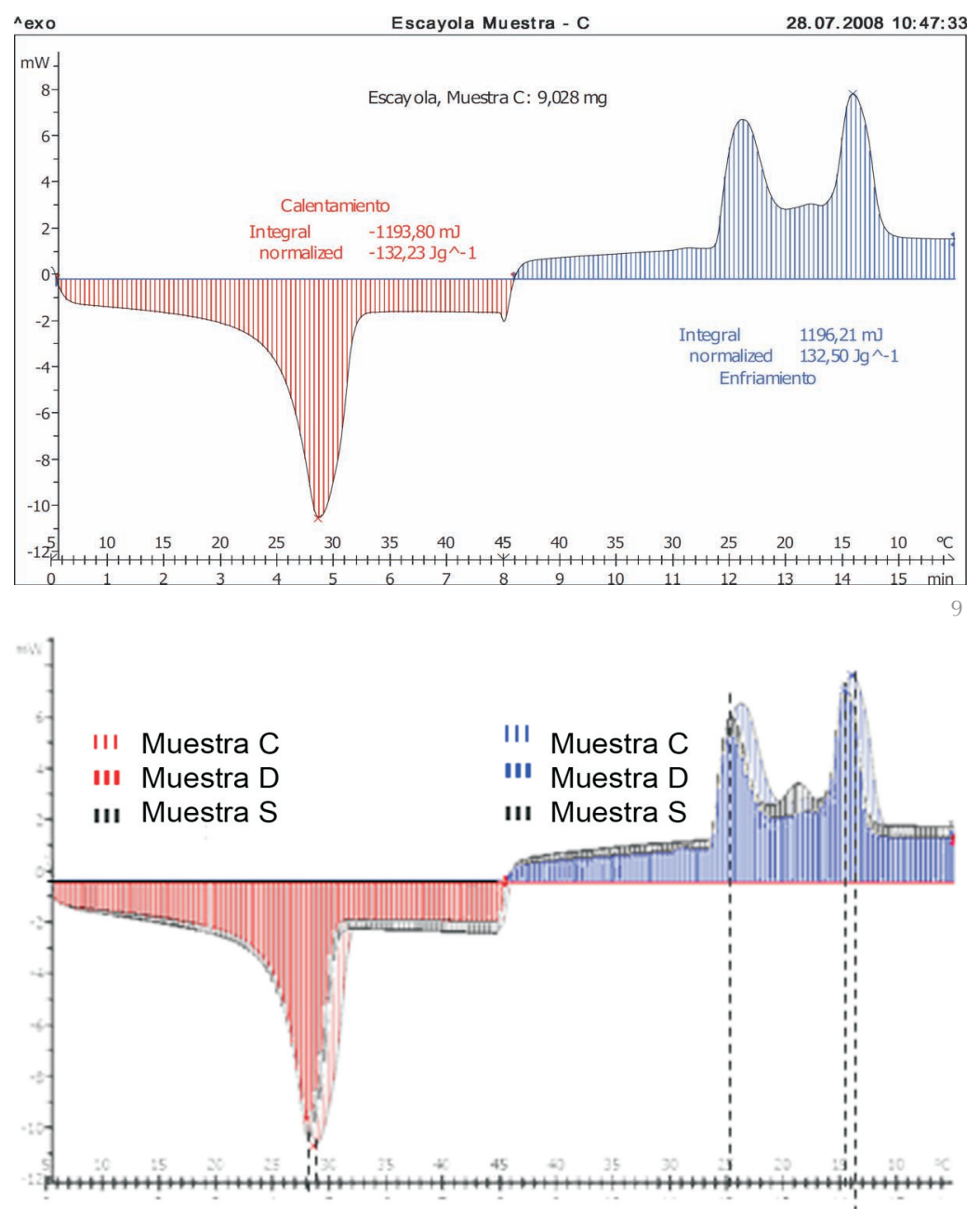

10. Comparación de las curvas de entalpía de las sustancias S, D y C.
La tasa de PCM que cambia de fase en el rango de temperaturas $18-35^{\circ} \mathrm{C}$, o tasa efectiva de PCM, está en torno al 80\%, como en el caso anterior.

\subsection{Muestra $C$}

La siguiente sustancia a analizar contiene la misma cantidad de PCM que de yeso.

Según el análisis para 9,028 mg de sustancia, se necesitan 1193,90 mJ, para elevar la tem- peratura de $0^{\circ}$ a $45^{\circ} \mathrm{C}$. Algo más se libera en el proceso de solidificación, 1.196,21 mJ, por lo que se puede suponer que a $45{ }^{\circ} \mathrm{C}$ cuando se inició el proceso de fusión una pequeñísima parte de sustancia estaba fundida.

Los valores obtenidos en este ensayo se expresan en la Tabla 5.

Se obtiene así que el calor sensible de la yeso y del PCM es de 1,472 y 2,054 mJ/g.K respectivamente. $\mathrm{Y}$ el calor latente de este último es de 105,792 mJ/g, muy próximo a los resultados obtenidos en los ensayos $\mathrm{S}$ y $\mathrm{D}$, de 106,328 y $104,024 \mathrm{~mJ} / \mathrm{g}(\mathrm{J} / \mathrm{g})$ respectivamente $(110 \mathrm{~J} / \mathrm{g}$, es el dato proporcioado por la empresa suministradora).

En esta sustancia la energía asociada por unidad de masa $\Delta \mathrm{H} / \mathrm{M}$ (para los $45^{\circ} \mathrm{C}$ ) de $132,233 \mathrm{~mJ} / \mathrm{g}$. Este valor es muy superior al obtenido en la sustancia Q (sin PCM), con 66,263 , y se ha incrementado proporcionalmente en los otros dos compuestos con 115,937 y $124,187 \mathrm{~mJ} / \mathrm{g}$ para las sustancias $S$ y D, respectivamente. Gráficamente se puede observar el mismo comportamiento reflejado en los diagramas anteriores, con una asimetría en los dos procesos, presentado la fase de solidificación dos picos. En este caso se han desplazado ligeramente respecto a los anteriores, produciéndose el primero a 23,5 ${ }^{\circ} \mathrm{C}$ y el segundo a $13,5^{\circ} \mathrm{C}$. (Figura 9).

El trazado de las curvas es exacto en los dos casos, la diferencia está en que en este caso la mayor cantidad de PCM en el compuesto produce una continuación de la curva, que representa la energía adicional necesaria para producir el cambio de fase.

Si se comparan las tres sustancias, S, D y C, con PCM, se obtiene la Gráfica 9, donde se podrán apreciar las ligeras diferencias entre ellas, pero de la que se puede extraer unas líneas generales de comportamiento (Figura 10).

Tras estos primeros análisis se sometieron las sustancias a un segundo ensayo con el DSC, cambiando las condiciones de contorno para verificar si el comportamiento que presentaban era intrínseco del material, o estaba influenciado por la velocidad y el rango de temperatura establecido en el primer test.

Así se procedió a realizar un segundo ensayo con las siguientes condiciones, cuyos resultados se exponen en la Figura 11:

- Sustancia D, ciclo de temperatura $5-40-5{ }^{\circ} \mathrm{C}$, a una velocidad de $2{ }^{\circ} \mathrm{C} / \mathrm{min}$ (como en los ensayos anteriores).

- Sustancia D, ciclo de temperatura 5-40-5 ${ }^{\circ} \mathrm{C}$, a una velocidad de $10^{\circ} \mathrm{C} / \mathrm{min}$. 
- Sustancia D, ciclo de temperatura 5-40-5 ${ }^{\circ} \mathrm{C}$, a una velocidad de $20^{\circ} \mathrm{C} / \mathrm{min}$.

Existe una amplia bibliografía sobre la interpretación de los resultados de ensayos con DSC, sobre la influencia de las condiciones de ensayo -principalmente la velocidad y el rango de temperatura-, en los resultados obtenidos. Algunas Tesis se centran en la caracterización de una sustancia a través de la interpretación de las gráficas obtenidas con el ensayo DSC de la misma. Esto es: difusividad térmica, conductividad, efusividad, calor específico, ...

Lo interesante para este trabajo, como se ha dicho anteriormente, es la obtención de la capacidad de almacenamiento térmico (sensible y latente), y el rango de temperaturas más efectivo de trabajo de la sustancia, es decir, donde el mayor porcentaje de ella sufre cambio de fase.

En este sentido se obtienen lo siguiente:

- Las gráficas obtenidas son concéntricas, registrándose la misma ventana térmica a diferentes velocidades de ensayo.

- El punto de inflexión -donde comienza a incrementarse la pendiente- en los tres casos es el mismo.

- Los resultados obtenidos son más acusados y notorios cuando el ensayo se realiza a mayor velocidad de cambio de temperatura, ya que el flujo aportado es mayor. Es por eso que con este método se utilizan velocidades de variación de temperatura del orden de $10-20^{\circ} \mathrm{C} / \mathrm{min}$.

Para verificar el comportamiento real de los paneles de yeso a una temperatura dada y con una variación de temperatura próxima a la real, se han ensayado en el laboratorio, y sus resultados se expondrán a continuación.

\subsection{Obtención de la ecuación}

Se ha seleccionado la gráfica-correspondiente al proceso de fusión a $20^{\circ} \mathrm{C} / \mathrm{min}$, de la muestra D (100Y-100A-3,5F-4D-80G) para obtener la ecuación de variación de la entalpía $\Delta \mathrm{H}(\mathrm{mW})$, en función de la temperatura $\left({ }^{\circ} \mathrm{C}\right)$. Se va a suponer el proceso de fusión y solidificación simétricos para mayor simplicidad.

Existen herramientas de cálculo especializadas en la simulación numérica para la obtención de la ecuación a partir de la gráfica, basadas en el Método de Diferencias Finitas, como TRNSYS, FORTRAN, ... En este caso, donde los valores obtenidos de la gráfica son discretos (cada 20 segundos) no es de interés utilizar esa metodología por lo que se ha utilizado la hoja de cálculo Excel, por su simplicidad de manejo, versatilidad y universalidad de uso.

Para obtener la ecuación se ha divido la función por tramos, que expresa la variación de la entalpía del compuesto por unidad de masa en función de la temperatura, es decir, la cantidad de PCM fundido, tal y como se representa en la Figura12. Para ello previamente se han
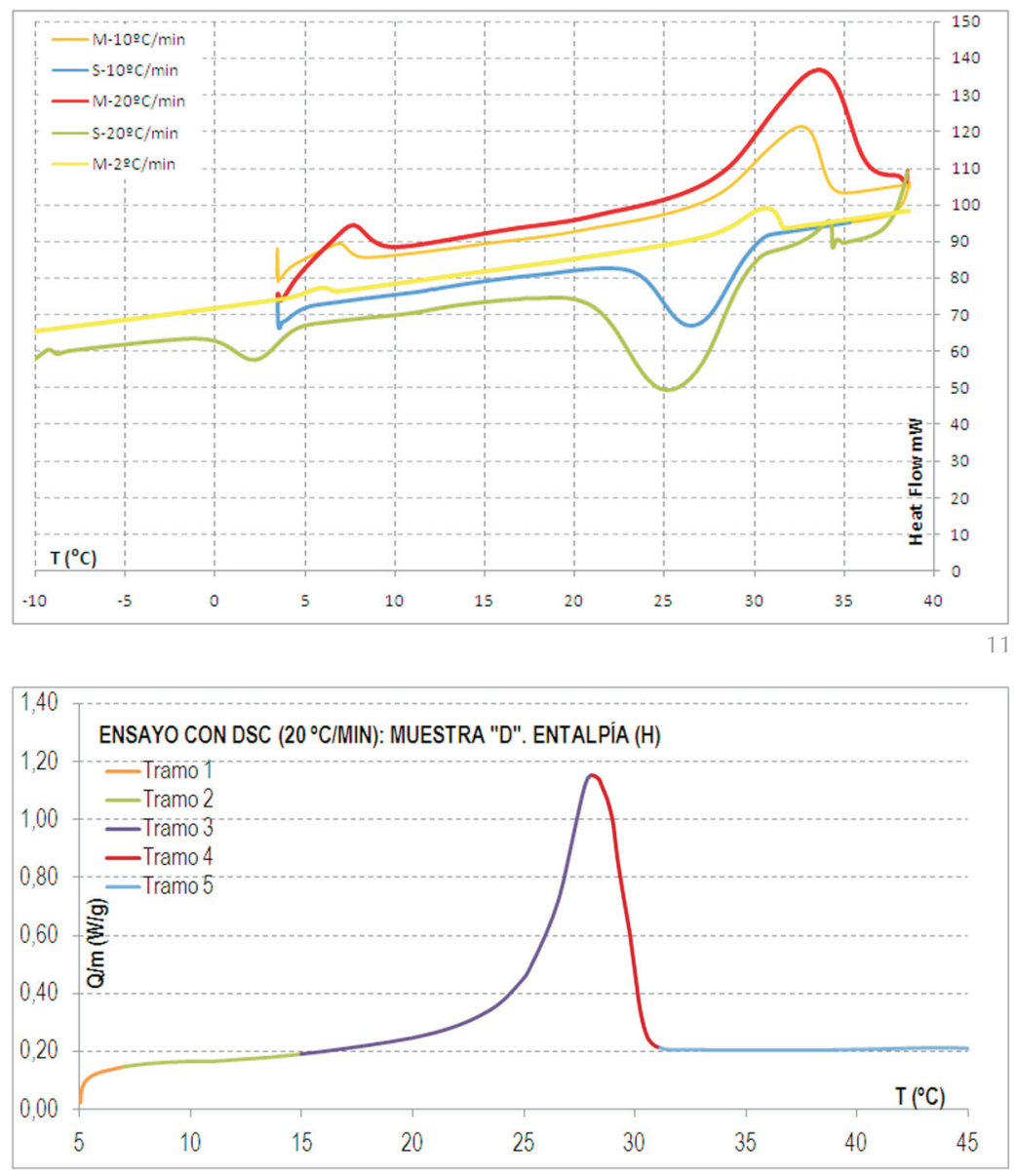

dividido los valores obtenidos de entalpía por la masa de la muestra, 8,237 mg.

Así podemos obtener las siguientes ecuaciones para los rangos de temperatura que son de interés en este trabajo $\left(15-35^{\circ} \mathrm{C}\right)$ [2]:

Tramo 1: $\left(5<\mathrm{T}<7{ }^{\circ} \mathrm{C}\right)$ No es temperatura utilizada en construcción

Tramo 2: $\left(7<\mathrm{T}<15^{\circ} \mathrm{C}\right)$ $\mathrm{H}=0,0048 \mathrm{~T}+0,1167$

Tramo 3: $\left(15<\mathrm{T}<28,5^{\circ} \mathrm{C}\right)$

$$
\mathrm{H}=0,0001 \mathrm{~T}^{4}-0,0094 \mathrm{~T}^{3}+
$$$$
0,2639 \mathrm{~T}^{2}-3,254 \mathrm{~T}+15,08
$$

Tramo 4: $\left(28,5<\mathrm{T}<31{ }^{\circ} \mathrm{C}\right)$

$\mathrm{H}=0,1049 \mathrm{~T}^{3}-9,3176 \mathrm{~T}^{2}+$ $275,34 \mathrm{~T}-2706,1$

Tramo 5: $\left(31<\mathrm{T}<45^{\circ} \mathrm{C}\right)$ $\mathrm{H}=0,0001 \mathrm{~T}+0,2055$ 
13. Energía almacenada en función de la temperatura $(\mathrm{J} / \mathrm{g} \cdot \mathrm{K})$.

14. Superposición de la gráfica de energía almacenada con la de porcentaje de PCM fundido en función de la temperatura.

15. Energía almacenada en función de la temperatura del material compuesto "D" por unidad de masa: calor sensible y latente.
Siendo H, la variación de entalpía o energía necesaria para la fusión del material por unidad de tiempo, $\mathrm{dH}(\mathrm{T}) / \mathrm{dt}$, expresada en $(\mathrm{mW})$ y $\mathrm{T}$ la temperatura del ensayo $\left({ }^{\circ} \mathrm{C}\right)$.

Podrían obtenerse ecuaciones en función de las características del material (transmitancia, calor específico en estado sólido, temperatura del horno de ensayo,...), como se ha propuesto en otras trabajos, pero ello complica la resolución del problema y se aleja del interés de esta Tesis.
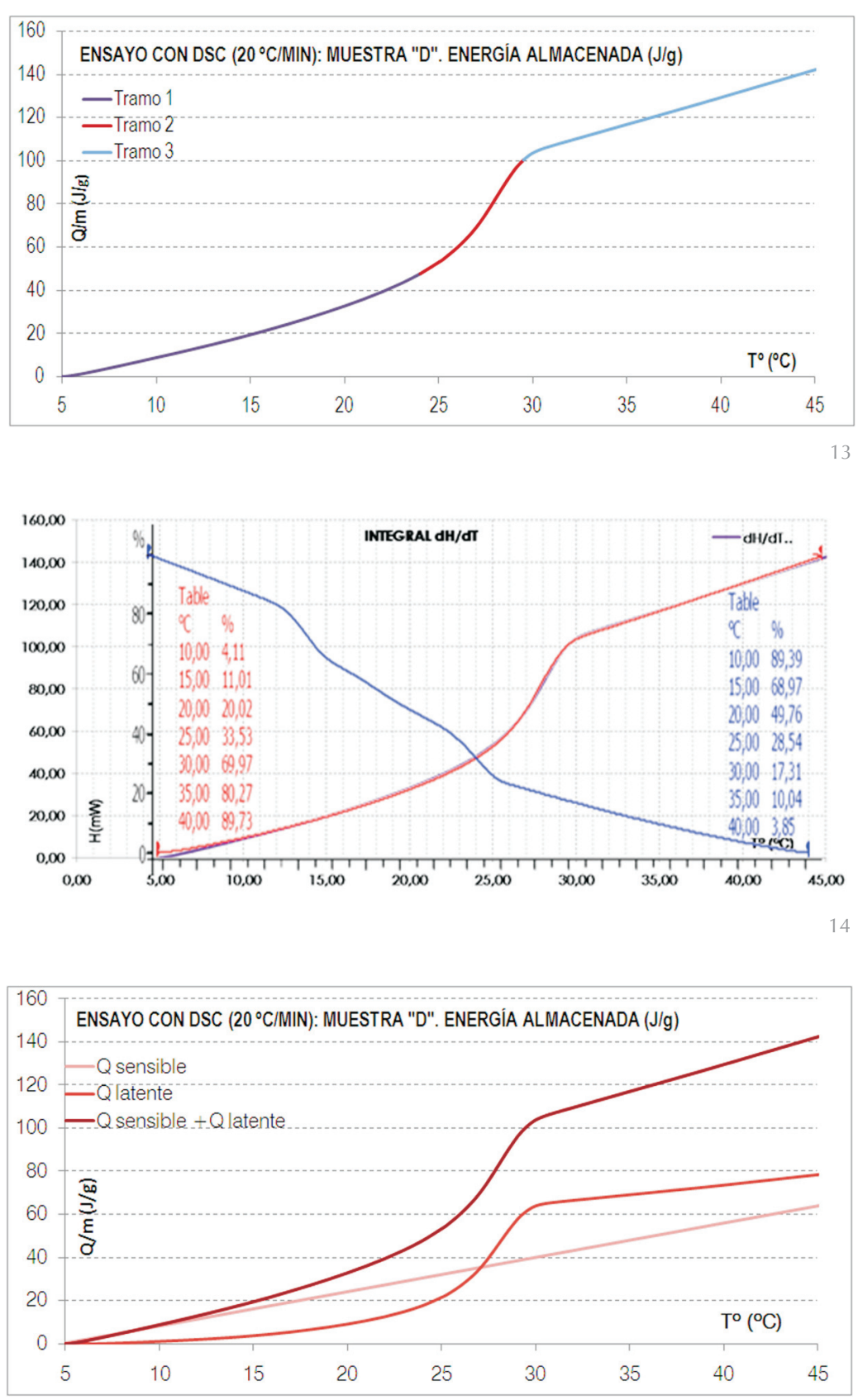

Operando con los valores obtenidos en la gráfica podemos obtener otra que exprese la cantidad de energía acumulada en cada tramo de temperatura, es decir la integral de la Figura 12, de acuerdo con la ecuación [3]:
Energía almacenada:

$$
\mathrm{H}(\mathrm{T})=\mathrm{Q}_{\mathrm{n}}=\mathrm{Q}_{\mathrm{n}-1}+\left(\mathrm{t}_{\mathrm{n}}-\mathrm{t}_{\mathrm{n}-1}\right) * \Delta \mathrm{H}_{\mathrm{n}^{\prime}}[3]
$$

Siendo $Q_{n}$ y $Q_{n-1}$ la energía acumulada en el tiempo $n, y$ - 1 respectivamente $(\mathrm{J} / \mathrm{g})$.

$t_{n} y_{n-1}$, el tiempo en la muestra $n, y n-1$ respectivamente, (s) y $\Delta \mathrm{H}_{n^{\prime}}$ la entalpía del material en la muestra $n,(m W)$.

Para facilidad de estudio se ha dividido en tramos. De cada uno se puede obtener una ecuación que relacione la cantidad de energía almacenada en cada intervalo (Figura13).

Si se superpone esta gráfica -la correspondiente a la energía almacenada para toda la muestra ensayada $(8,237 \mathrm{mg})-$, a la de porcentaje de material de cambio de fase fundido en función de la temperatura, observamos la gran coincidencia entre ambas: Figura 14.

Su trazado es la suma de dos gráficas, la correspondiente al calor sensible del material compuesto $\left(\mathrm{m}_{\mathrm{y}}{ }^{*} \mathrm{Ce}_{\mathrm{y}}+\mathrm{m}_{\mathrm{pcm}}{ }^{*} \mathrm{ce}_{\mathrm{pcm}}\right)$, la del calor latente del mismo $\left(\mathrm{m}_{\mathrm{pcm}}{ }^{{ }^{*}} \mathrm{clat}_{\mathrm{pcm}}{ }^{\mathrm{pcm}}\right)$. En la Figura 15 se representan las tres funciones: calor sensible, calor latente y entalpía.

La primera es de carácter lineal, con lo cual solo añade a la gráfica suma su pendiente constante.

La segunda, que expresa el almacenamiento de energía en forma de calor latente, es la que influye de manera más significativa en el trazado de la gráfica suma. Y depende exclusivamente de la cantidad de PCM fundido en cada intervalo de temperatura.

A partir de estos datos podemos obtener los valores para un panel realizado con este material compuesto de $1,0 \mathrm{~m}^{2}$ y $1,5 \mathrm{~cm}$ de espesor, el cual contiene 4,754 kg de PCM y 5,935 kg de yeso.

La ecuación obtenida para cada gráfica es [3]:

$$
\begin{gathered}
\mathrm{Q}_{\text {sens }}: \Delta \mathrm{H}=16,72 \mathrm{~T}-83,6 \\
\mathrm{Q}_{\text {lat }}=\operatorname{Tramo~} 1: \mathrm{T}<17^{\circ} \mathrm{C}, \\
\Delta \mathrm{H}=20,856 \mathrm{~T}-109,75
\end{gathered}
$$

Tramo 2: $17 \leq \mathrm{T}<30$ :

$$
\begin{aligned}
& \Delta \mathrm{H}=-0,064 \mathrm{~T}^{4}+6,3653 \mathrm{~T}^{3}- \\
& 228,74 \mathrm{~T}^{2}+3581,6 \mathrm{~T}-20461
\end{aligned}
$$

Tramo 3: $\mathrm{T} \geq 30$ :

$$
\Delta \mathrm{H}=26,224 \mathrm{~T}+304,74
$$

Para tener una noción más clara de la cuantía de estos valores de capacidad de almacenamiento térmico se van a comparar con dos sistemas constructivos utilizados también en el revestimiento interior de paramentos verti- 
cales: placa de yeso laminado de $1,5 \mathrm{~cm}$ de espesor, y $1 \frac{12}{2}$ pie de fábrica de ladrillo hueco, los cuales se representan en la Figura 16.

Integrando la función $\mathrm{dH} / \mathrm{dT}$, de cada material se obtiene la expresión de energía acumulada por cada uno de ellos en cada momento. Las funciones se representan en la Figuta 17.

Los valores representados en la Figura 17 se exponen en la Tabla 6. En ella se consideran los valores comprendidos en el rango de temperaturas $20-30{ }^{\circ} \mathrm{C}$, que es el utilizado en edificación por estar en torno a la temperatura de confort.

Relacionando entre sí los valores de la Tabla 6 se obtiene la Tabla 7. Se ha considerado para el compuesto de yeso y material de cambio de fase una densidad de $725 \mathrm{~kg} / \mathrm{m}^{3} ; 800 \mathrm{~kg} / \mathrm{m}^{3}$ para la del yeso y $1600 \mathrm{~kg} / \mathrm{m}^{3}$ para la cerámica (fábrica de ladrillo hueco).

\section{CONCLUSIONES}

Del análisis de las propiedades térmicas intrínsecas del compuesto -curva de entalpía asociada a la temperatura-se concluye que es propia de un PCM orgánico (parafina) con las siguientes características:

- Asimetría en el almacenamiento de calor latente en los procesos de fusión y solidificación (relación entalpía-temperatura) así como asimetría e irregularidad (no linealidad) de las gráficas de porcentaje de fusiónsolidificación (expresado de 0 a 100\%).

- Amplia ventana térmica, (entre $8,24^{\circ} \mathrm{C}$, y $35,18^{\circ} \mathrm{C}$ ) con mayor proporción de cambio de fase entre $20-30^{\circ} \mathrm{C}$.

- El calor sensible y latente del compuesto es de 2,054 J/g ${ }^{\circ} \mathrm{C}$ y $106,32 \mathrm{~J} / \mathrm{g}$, en el rango de temperatura estudiado $\left(5-45^{\circ} \mathrm{C}\right)$.

- La curva de energía almacenada (J) en el tiempo es idéntica a la de porcentaje de material que cambia de fase en el tiempo.

Se puede determinar una expresión matemática sencilla que relacione la entalpía de la sustancia y la temperatura, para determinar su comportamiento térmico, la cual puede compararse con cualquier otro material constructivo. Ec[4], (Figura 18).

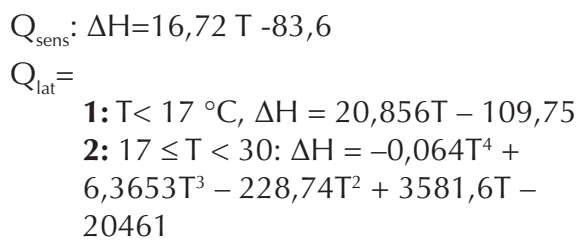

La capacidad de almacenamiento térmico por unidad de masa de un compuesto de yeso y PCM $(44,5 \%)$ es más de 5 veces superior a la del yeso, y 8 veces superior a la de la cerámica -fábrica de ladrillo hueco- en el rango de temperatura $\left(25-30^{\circ} \mathrm{C}\right)$. Esta relación medida en energía por unidad de volumen es de 4,7 veces la capacidad de almacenamiento del yeso y 4,2 veces la de la cerámica, en el mismo rango de temperatura.
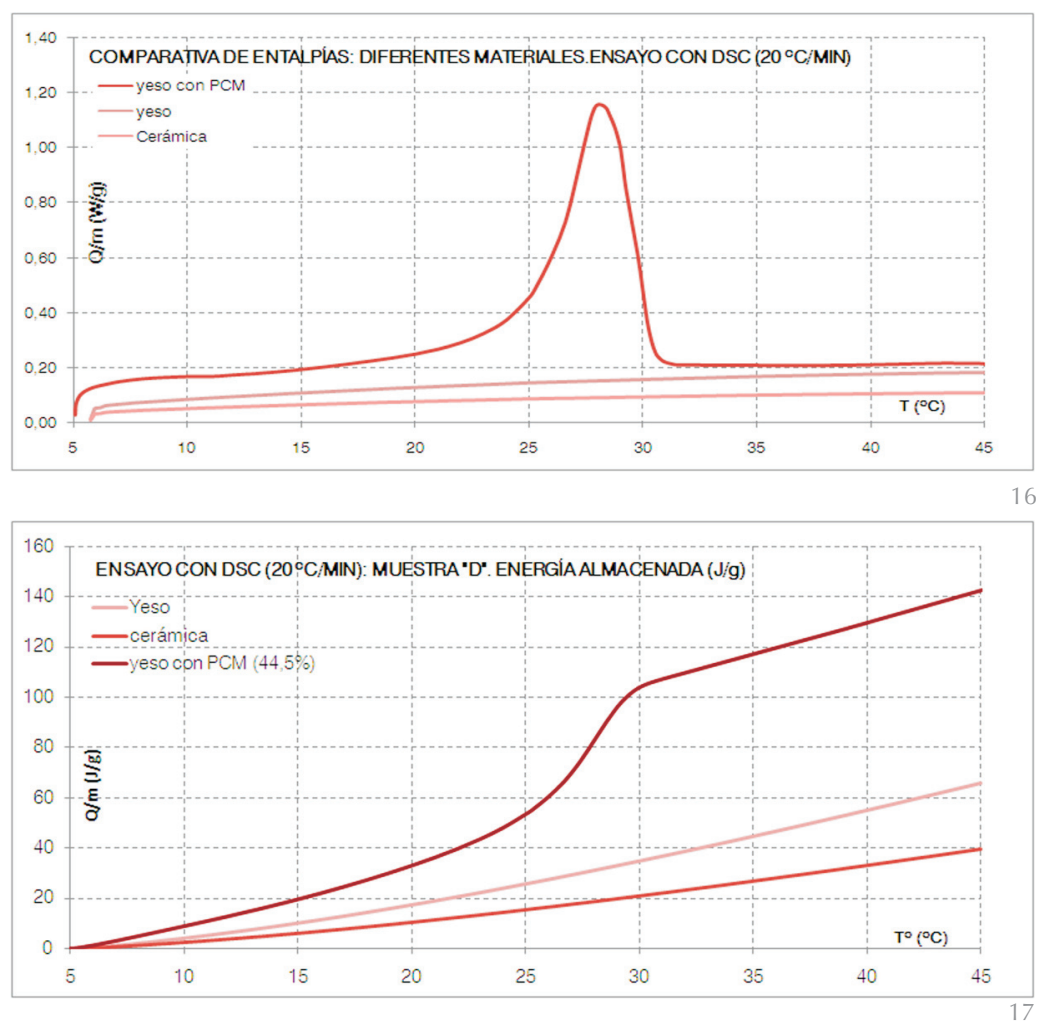

Tabla 6

Comparativa de la capacidad de almacenamiento térmico por unidad de masa y unidad de volumen de tres materiales distintos en el rango de temperaturas utilizado en edificación $\left(20-30{ }^{\circ} \mathrm{C}\right)$

\begin{tabular}{|l|c|c|c|c|}
\hline & \multicolumn{2}{|c|}{$\begin{array}{c}\text { Energía almacenada por } \\
\text { unidad de masa }\end{array}$} & \multicolumn{2}{c|}{$\begin{array}{c}\text { Energía almacenada por } \\
\text { unidad de volumen }\end{array}$} \\
\hline Rango de To & $20-25{ }^{\circ} \mathrm{C}$ & $25-30{ }^{\circ} \mathrm{C}$ & $20-25^{\circ} \mathrm{C}$ & $25-30{ }^{\circ} \mathrm{C}$ \\
\hline MATERIAL & $\mathrm{J} / \mathrm{g}$ & $\mathrm{J} / \mathrm{g}$ & $\mathrm{kJ} / \mathrm{m}^{3}$ & $\mathrm{~kJ} / \mathrm{m}^{3}$ \\
\hline Yeso & 8,11 & 9,73 & $6.484,68$ & $7.780,75$ \\
\hline Cerámica & 4,86 & 5,84 & $6.808,91$ & $8.169,79$ \\
\hline Yeso + PCM & 20,38 & 50,42 & $14.773,63$ & $36.551,93$ \\
\hline
\end{tabular}

Tabla 7

Relación de la capacidad de almacenamiento térmico por unidad de masa y unidad de volumen de yeso y cerámica comparados con compuesto de PCM y yeso $\left(\mathrm{T}^{\circ}=20-30^{\circ} \mathrm{C}\right)$

\begin{tabular}{|l|c|c|c|c|}
\hline & \multicolumn{2}{|c|}{$\begin{array}{c}\text { Energía almacenada por } \\
\text { unidad de masa }\end{array}$} & \multicolumn{2}{c|}{$\begin{array}{c}\text { Energía almacenada por } \\
\text { unidad de volumen }\end{array}$} \\
\hline Rango de T & $20-25{ }^{\circ} \mathrm{C}$ & $25-30{ }^{\circ} \mathrm{C}$ & $20-25{ }^{\circ} \mathrm{C}$ & $25-30{ }^{\circ} \mathrm{C}$ \\
\hline MATERIAL & $\mathrm{J} / \mathrm{g}$ & $\mathrm{J} / \mathrm{g}$ & $\mathrm{kJ} / \mathrm{m}^{3}$ & $\mathrm{~kJ} / \mathrm{m}^{3}$ \\
\hline yeso & 2,51 & 5,18 & 2,28 & 4,70 \\
\hline cerámica & 4,19 & 8,64 & 2,17 & 4,47 \\
\hline yeso + PCM & 1,00 & 1,00 & 1,00 & 1,00 \\
\hline
\end{tabular}


18. Energía almacenada por el material compuesto: calor sensible y latente.
Con ello se concluye que la incorporación de material de cambio de fase a un material constructivo como el yeso, mejora considerablemente su capacidad de almacenamiento térmico -inercia térmica-, que, combinado con estrategias pasivas, ayuda a reducir las cargas de calefacción y refrigeración de un edificio.

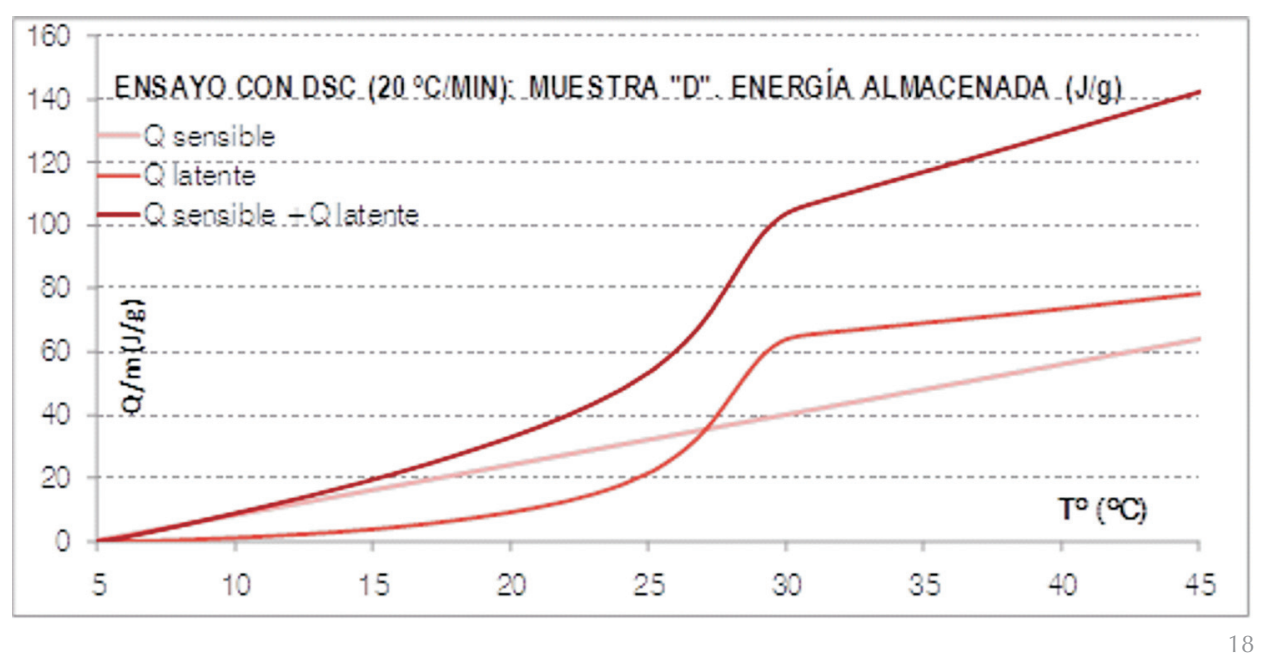

\section{BIBLIOGRAFÍA}

(1) Abhat, A.: Low temperature latent heat thermal energy storage-Heat storage materials. Solar Energy, 1983. 30(4).

(2) Khudhair, A.M.; Farid, M.M.: "A review on energy conservation in building applications with thermal storage by latent heat using phase change materials". Energy Conversion and Management, 2004. 45(2): p. 263-275.

(3) Feldman, D.; Banu, D.: DSC analysis for the evaluation of an energy storing wallboard. Thermochimica acta, 1996. 272: p. 243-251.

(4) Hawes, D.; Feldman, D.; Banu, D.: "Latent heat storage in building materials". Energy and buildings, 1993. 20(1): p. 77-86.

(5) Hawlader, M.; Uddin, M.; Khin, M.: "Microencapsulated PCM thermal-energy storage system". Applied energy, 2003. 74(1-2): p. 195-202.

(6) Collier, R.K.; Grimmer, D.P.: Experimental evaluation of phase change material building walls using small passive test boxes. 3. national passive solar conference, 1979. 11.

(7) Salyer: Dry Powder Mixes comprising Phase Change Materials. 1993: US.

(8) Zalba, B.; et al:: "Review on thermal energy storage with phase change: materials, heat transfer analysis and applications". Applied Thermal Engineering, 2003. 23(3): p. 251-283.

(9) Shapiro, M.; et al.: Thermal storage in drywall using, organic phase-change material. Passive Sol. J, 1987. 4(4).

(10) Gibbs B, H.S.: DSC study of technical grade phase change heat storage materials for solar heating applications. in International Solar Energy Conference. ASME/JSME/JSEJ. 1995.

(11) Feldman, D.; Banu, D.; Hawes, D.W.: "Development and application of organic phase change mixtures in thermal storage gypsum wallboard". Solar Energy Materials and Solar Cells, 1995. 36(2): p. 147-157.

(12) Athientis, A.K.; L.C.; Hawes, D.; Banu, D.; Feldman, D.: "Investigation of the thermal performance of a passive solar test-room with wall latent heat storage", in Building and Environment. 1997. p. 405-410.

(13) Darkwa, K.; Kim, J.: "Dynamics of energy storage in phase change drywall systems". International Journal of Energy Research, 2005. 29(4): p. 335-343.

(14) Rudd, A.F.: Phase-change material wallboard for distributed thermal storage in buildings. ASHRAE Transactions, 1993. 99(2): p. 339-46.

(15) Scalat, S.; et al.: "Full scale thermal testing of latent heat storage in wallboard". Solar Energy Materials and Solar Cells, 1996. 44(1): p. 49-61.

(16) De Grassi, M.; Carbonari, A.; Palomba, G.: "A statistical approach for the evaluation of the thermal behavior of dry assembled PCM containing walls". Building and Environment, 2006. 41(4): p. 448-485.

(17) Heim, D.; Clarke, J.A.: "Numerical modelling and thermal simulation of PCM-gypsum composites with ESP-r". Energy \& Buildings, 2004. 36(8): p. 795-805.

(18) Neeper, D.A.: "Thermal dynamics of wallboard with latent heat storage". Solar Energy, 2000. 68(5): p. 393403.

(19) Kang, Y.H.; Kwak, H.Y.; et al.: Numerical heat transfer analysis of heat storage board with microcapsule using phase change material. 1997.

(20) García-Santos, A.: "Caracterización de compuestos de escayola reforzados, en relación con el tipo de refuerzo y la relación a/y". Informes de la Construcción, 2004. 56: p. 19-31.

(21) Oliver, A.: Incorporación de Materiales de Cambio de Fase en Placas de Yeso Reforzadas con Fibras de Polipropileno. Aplicación a Sistemas de Calefacción y Refrigeración Pasivos para Alamcenamiento de Calor Latente en Edificios; in Dpto. de Construcción y Tecnología Arquitectónicas. 2009, Universidad Politécnica de Madrid: Madrid. p. 376. 